\title{
Optimization of Authorised/Licensed Shared Access resources
}

\author{
Eva Pérez, Karl-Josef Friederichs, \\ Andreas Lobinger, Simone Redana \\ Nokia Solutions and Networks $\mathrm{GmbH}$ \\ Munich, Germany \\ Email:\{eva.perez, karl-josef.friederichs, \\ andreas.lobinger, simone.redana\}@nsn.com
}

\author{
Ingo Viering, Juan Diego Naranjo \\ Nomor Research $\mathrm{GmbH}$ \\ Munich, Germany \\ Email: \{viering,naranjo\}@nomor.de
}

\begin{abstract}
The expected increase of the traffic volume in mobile broadband networks and the upcoming spectrum scarcity, require an optimization of the usage of the spectrum. One of the solutions is Authorised/Licensed Shared Access (ASA/LSA) ${ }^{1}$, a new regulatory concept that allows license holders (incumbents) to share spectrum with other service providers, under welldefined conditions, unlocking additional bands which are used only partially in time and/or location. This paper shows the benefits of ASA, considering different methods to optimize the resources, by simulating an LTE network where a Mobile Network Operator (MNO) is allowed to use the $2300 \mathrm{MHz}$ band as an ASA licensee.
\end{abstract}

Keywords-component; Authourised Shared Access (ASA); Licensed Shared Access (LSA), spectrum efficiency

\section{INTRODUCTION}

Mobile and wireless communication networks will need to cope with the tremendous increase in data traffic anticipated over the next decade. Spectrum can be looked upon as the real estate for Mobile Broadband (MBB) in addressing this challenge. Beyond the levers of increased network densification and enhanced spectral efficiency more radio spectrum is clearly needed for mobile networks to fulfil capacity and coverage demands [1]. In Europe, a total of around $600 \mathrm{MHz}$ of spectrum is currently allocated to MBB, and significantly more additional spectrum will be needed towards 2020. Spectrum between $400 \mathrm{MHz}$ and $6 \mathrm{GHz}$ is best suited for mobile applications as lower bands would require antennas too large to be integrated into mobile devices, and higher bands would limit cell sizes. This entire range of good spectrum, however, is already allocated to a number of different services and technologies, such as broadcast, aeronautical, satellite, defence, public safety and other commercial and non-commercial services; many of which do not utilise the spectrum intensively.

In some cases spectrum sharing may be, cost-wise and time-wise, a very efficient mean to gain at least partial access to additional spectrum resources for $\mathrm{MBB}$ use. Mobile networks target to offer predictable quality of service; therefore

${ }^{1}$ The Radio Spectrum Policy Group (RSPG) and the European Commission largely adopted and generalized the concept but renamed it to 'Licensed Shared Access' where ASA is framed within LSA it is required that sufficient control mechanisms be implemented when applying spectrum sharing. Authorized Shared Access (ASA), also known as Licensed Shared Access (LSA) provides a solution for bands that cannot easily be refarmed or totally vacated by their incumbent users, but where actual spectrum usage is underutilized and infrequent [2] [3] [3].

Through this new access model a primary license holder (incumbent) would grant spectrum access rights to one or more other users which may then use the band under specific service conditions. Conditions defining how the spectrum may be used would be subject to individual agreements, and to permission from the National Regulatory Authority (NRA). The NRA would be expected to issue licenses to one, or a very limited number of mobile operators that would allow them to use specific bands as ASA licensees. Thereby orthogonal usage by time or location should always be coordinated between the operators and the incumbent in order that a high level of service performance and predictability can be realised.

The ASA licensee should be responsible for compliance with technical requirements obtained from the incumbent such as meeting certain interference thresholds. This can best be accomplished via an ASA Controller under the full control of the network operator.

In consideration of concrete ASA frequencies the 2300$2400 \mathrm{MHz}$ band is especially attractive because it is already an IMT band recommended by the ITU-R and is in use in some countries particularly in Asia Pacific regions.

The results presented in this paper are focused on the 2300$2400 \mathrm{MHz}$ band as additional spectrum for LTE which can be made available also in certain European countries thanks to ASA. However, there are different wireless systems allowed to use this band: SAP/SAB video links, Telemetry, Unmanned Aircraft Systems (UAS) or Amateur services may be using this spectrum band [4]. Since the characteristics of each system are different (user position, height, maximum interference allowed, etc), the requirements could be different depending on the incumbent. Considering different examples of incumbent spectrum users, this paper shows the benefits of using the ASA resources for an LTE system, including options for an optimization of the ASA resources. 


\section{ASA SPECTRUM MANAGEMENT SIMULATION}

The ASA functionality has been included in a dynamic system level simulator, which evaluates certain KPIs, such as load or throughput, at individual cells and individual users. This section will describe the scenario and the mechanisms to detect and clear the interference from the LTE system to the incumbent.

\section{A. Simulation scenario}

The scenario consists of 19 LTE sites (Fig. 1), each of them with three sectors, where initially the MNO uses the $800 \mathrm{MHz}$ frequency band. Additionally, the MNO is an ASA Licensee, being allowed to use the $2300 \mathrm{MHz}$ band (ASA resources). However, there is a Reservation Area (RA), i.e., area where the incumbent may use the spectrum occasionally. Whenever the incumbent uses the spectrum at the RA, the MNO will need to evacuate the area to not interfere the incumbent spectrum users.

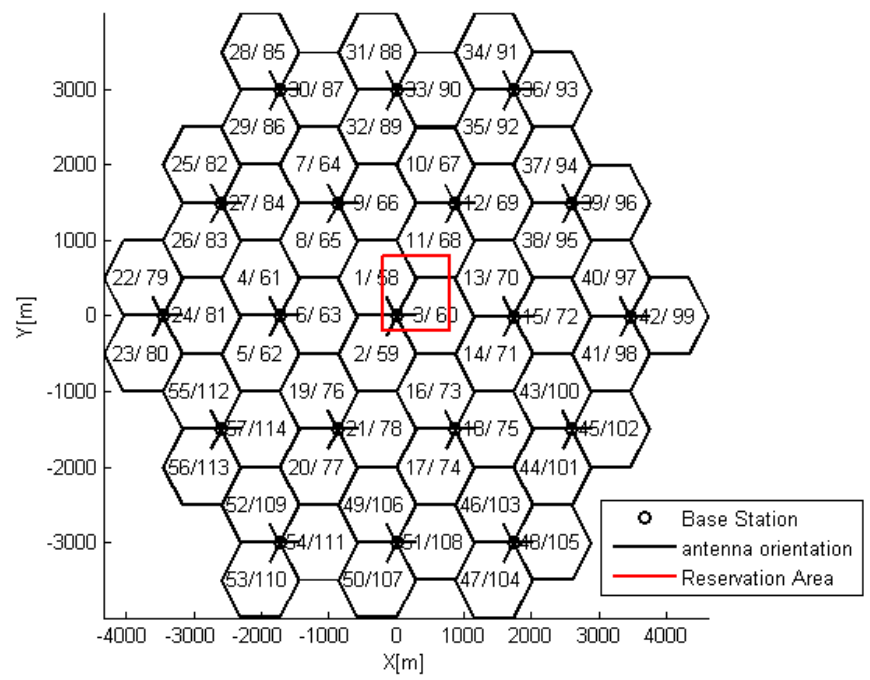

Fig. 1. Simulation scenario

The simulation considers a significantly loaded scenario: initially, the simulation starts with 90 users connected per site, transmitting packets of $5 \mathrm{MB}$ on average and average arrival rate of 5s. Although Carrier Aggregation (CA) could be used to benefit from the additional carrier, it has not been considered in this case due to the uncertainty of how many CA-capable UEs may be in the network. Instead of CA, the MNO will use Traffic Steering at Connection Setup to share the load with the additional carrier, i.e., cells highly loaded steer users starting a new connection to the other layer [5] [6].

\section{B. Interference detection}

The MNO should fulfil technical requirements obtained from the incumbent such as meeting certain interference thresholds. However, the aim of the MNO is to provide the best service to their customers. In order to maximize MNO's coverage in the ASA layer without interfering the incumbent, the MNO will have to detect the limits where the spectrum can be used.

The simulations are considering that the incumbent is interfered if the MNO exceeds the interference criterion (IC) inside the RA, i.e. the maximum interference allowed. The simulation is considering two methods to detect interfering cells. After the detection, these cells will be shutdown or modified to clear the interference.

The first method, considers the measurements from users. Similarly to the feature Minimization of Drive Test (MDT), the measurement reports and location information from the users are collected. If the user is inside the RA, and the measurement report reveals that the Received Signal Received Power (RSRP) is higher than the IC, the cell where the user is connected will be detected as interfering.

In mathematical words, a cell $i$ is denoted as interfering cell if any user $u$ located in the RA reports an RSRP towards this cell $\mathrm{i}$ above the IC:

$$
\operatorname{RSRP}(\mathrm{i}, \mathrm{u})>\mathrm{P}_{\mathrm{IC}}
$$

Since MNO users may not be located at all locations where the incumbent spectrum users are, and where the MNO may also interfere, a second method is considered additionally. This second method consists of a set of test points $\overrightarrow{\mathrm{x}}_{\mathrm{k}}$ distributed inside the RA. The received signal $P_{R x}\left(j, \vec{x}_{k}\right)$ from each cell $j$ is estimated on each of these test points. If the received signal from a cell is exceeding the IC on a test point, that cell is detected as interfering.

In mathematical words, a cell $\mathrm{j}$ is denoted as interfering cell if any test point $\vec{x}_{k}$ violates the IC:

$$
P_{R x}\left(j, \vec{x}_{k}\right)>\mathrm{P}_{\mathrm{IC}}
$$

In reality it is not obvious how to obtain the interference levels $P_{R x}\left(j, \vec{x}_{k}\right)$. It will be difficult to extrapolate them from MDT data. The most precise but also most expensive option would be to install probes at the test points or to equip the incumbent's devices with measurement capabilities. Alternatively we could try to approximate them by propagation models which is simple but will suffer from inaccuracy.

In the simulation we use propagation models to calculate the levels, and for the sake of simplicity we assume that the levels are well known (genie approach). Note that the propagation models may differ from those usually employed by MNOs, as discussed later on. But the structure is the same:

$$
P_{R x}\left(j, \vec{x}_{k}\right)=P_{T x}(i)-P L\left(j, \vec{x}_{k}\right)-A\left(j, \vec{x}_{k}\right)+S\left(j, \vec{x}_{k}\right)
$$

where $P_{R x}(j, k)$ is the received power from the cell $j$ at the test point $\mathrm{k}, \mathrm{PL}$ is the path loss, which will depend on the propagation model (3GPP or Free Space), $A(j, k)$ are the antenna directivity losses, due to elevation and also azimuth, and $\mathrm{S}(\mathrm{j}, \mathrm{k})$ is the shadowing.

Since the incumbent users may not be located necessarily at the ground level, two different layers of test points are considered: one at ground level, and a second one at a higher position.

Whenever any of the two mechanisms detects a potential interference, some measures (described in sections D and E) will be needed to clear the interference from these cells. 


\section{Antenna model}

There is a different range of potential users at the 2300$2400 \mathrm{MHz}$ band: Video Links, Telemetry, Unmanned Aircraft Systems, other Broadband Wireless Systems, or Amateur Service [4]. Each one of these users may have different locations: from ground floor, such a wireless camera, to thousands of meters, such an unmanned aircraft. Additionally, each type of receptor may tolerate a different level of interference.

The location of the user of the incumbent spectrum must be differentiated when estimating the received power. There are three aspects which will have to be considered: path loss, shadowing and antenna pattern.

Depending on the location of the user or test point the propagation model considered for the simulations is different: if the position is at ground level, the 3GPP propagation model is used [7], if the position is above the buildings, the free space propagation model is used, and finally if the position is above the ground level, but not above the buildings, a model based on the Okumura Hata model for urban area is used [8].

In the case of users or test points below building height, the shadowing component is added to the received signal, while in the case of users or test points above buildings; there will not be a shadowing component, since there are no reflections from buildings, trees, cars, etc.

Fig. 2 shows a real antenna pattern and the antenna pattern model used for the simulations. If the receiver is at positions below the top of the building, the antenna nulls are smoothed out since in all angles it may get reflections from stronger lobes of the diagram. In this case $\left(0^{\circ}\right.$ to $180^{\circ}$ respect to the antenna), the directivity losses are obtained using the antenna pattern described in described in [9]:

$$
\begin{gathered}
A_{H}(\varphi)=-\min \left[12\left(\frac{\varphi}{\varphi_{3 d B}}\right)^{2}, A_{m}\right] \\
\varphi_{3 d B}=70 \text { degrees, } A_{m}=25 \mathrm{~dB} \\
A_{V}(\theta)=-\min \left[12\left(\frac{\theta-\theta_{3 d B}}{\theta_{3 d B}}\right)^{2}, S L A_{v}\right] \\
\theta_{3 d B}=10 \text { degrees, } S L A_{v}=20 \mathrm{~dB} \\
A(\varphi, \theta)=-\min \left\{-\left[A_{H}(\varphi)+A_{V}(\theta)\right], A_{m}\right\}
\end{gathered}
$$

where $\varphi, \theta$ are the horizontal and vertical angles from the test points to the antenna, respectively, and $A_{m}, S L A_{v}$ are the maximum directivity loss in the vertical plane and horizontal plane respectively.

While this model is well suited for these positions, it may not be applicable for test points in a large height $\left(180^{\circ}\right.$ to $360^{\circ}$ respect to the antenna, in Fig. 2). For these scenarios, the model has been modified: it consists of a normal distribution, based on the mean and standard deviation observed at a real pattern.

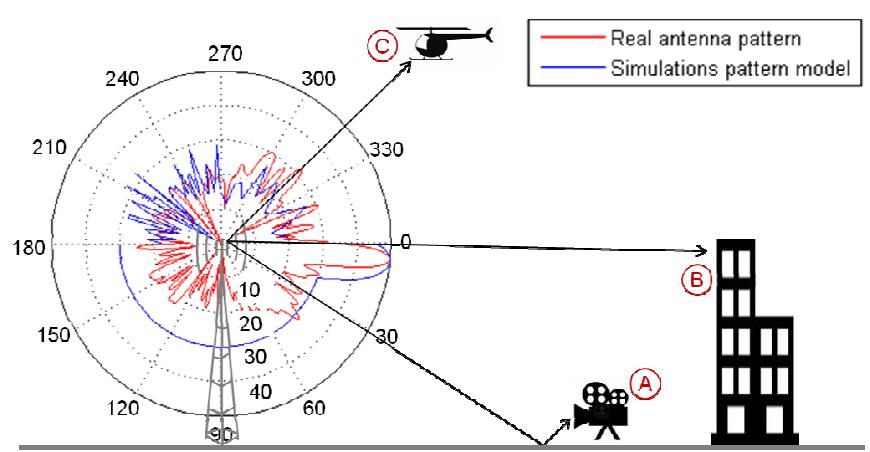

Fig. 2. Antenna model

\section{Interference clearance}

Once the interference has been detected, the MNO must clear it. The initial and simplest mechanism is to shutdown the interfering cell. However, this solution may not be the most efficient, especially from the point of view of the MNO, and the impact to the users.

In order to reduce the number of cells that need to be shutdown, increasing the coverage and minimizing the impact on the users, alternative mechanisms can be used to clear the potential interference in the RA.

The mechanisms described in this paper consist on reducing the transmitted power ( $\mathrm{Tx}$ power) of the interfering cells located in the surrounding areas of the RA, or modifying the downtilt of the cell, moving the main beam outside of the RA.

\section{E. Border optimization mechanisms}

This paper describes four different methods to clear the interference from the RA: two of them are based on the TX power reduction, and another two are based on the downtilt increase.

The question is, how much the Tx power should be reduced or how much the cell should be down tilted. For each case, two different methods have been considered. The first one, will consist on a pre-defined value for all interfering cells, and a final revision of the interference after the modification, shutting down the cells where the modification is not sufficient to clear the interference. The second method finds out, independently for each cell, how much the transmitted power should be decreased, or the downtilt increased, to clear the interference from the RA.

Combining the type of modification, Tx power or downtilt, and how this modification is done (fix or adapted), these are the four methods:

- $\quad$ Fixed Power step Optimization (FPO)

- $\quad$ Adapted Power step Optimization (APO)

- $\quad$ Fixed Tilt step Optimization (FTO)

- $\quad$ Adapted Tilt step Optimization (ATO) 


\section{RESULTS}

The simulations have considered three different types of incumbent spectrum users (Fig. 2): wireless cameras (ground level), cameras on buildings $(25 \mathrm{~m})$ and video links (mounted on helicopters, 150m) [10].

Considering the scenario (Fig. 1) and the interference detection mechanisms described in the previous section, the following results show the number of cells shutdown or modified, the average user throughput, and the coverage maps. For all cases, it has been considered an Interference Criterion of $-95 \mathrm{dBm}$.

\section{A. Wireless cameras}

Depending on the border optimization mechanism described, the number of MNO cells that allowed to be on is different. In this scenario, 16 cells are not interfering, being allowed to use the ASA spectrum without any modification, and 41 cells are detected as interfering. These 41 cells need to be shutdown or modified.

Considering that the default value for the Tx power of all cells is $46 \mathrm{dBm}$, Fig. 3 shows the number of cells using the ASA resources which allowed to be on when the Tx power value is modified.

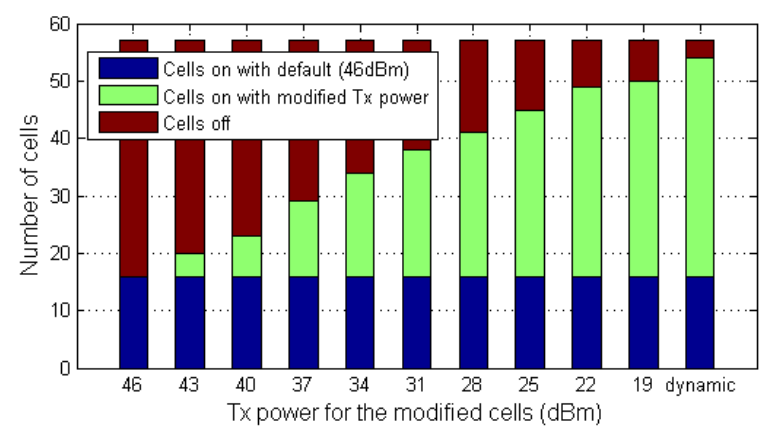

Fig. 3. Txpower optimization for wireless cameras: Number of cells on, modified and off

In the case of FPO, different Tx power values have been used (from the default $46 \mathrm{dBm}$ up to $19 \mathrm{dBm}$ ), which are applied to all cells, while in the case of the APO, different cells have different Tx power depending on the interference to the RA (Fig. 4).

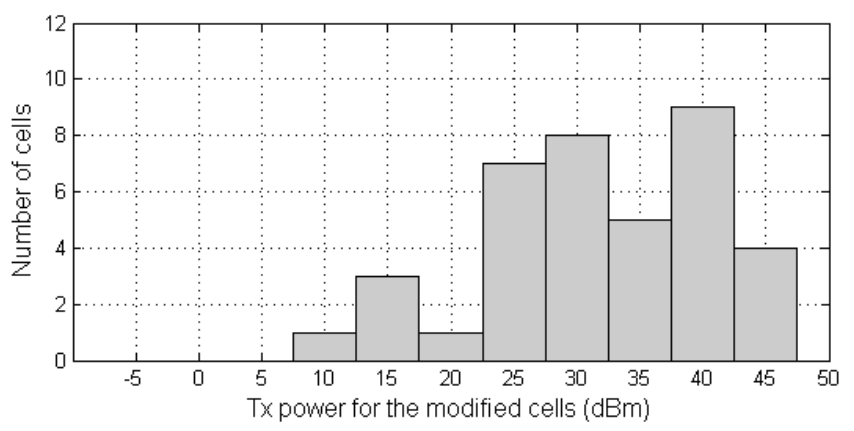

Fig. 4. Histogram of the Tx power for APO (wireless cameras)
When FPO Tx power is lower, the number of cells allowed to be on is higher, and when higher FPO Tx power values are used, more cells need to be shutdown since the Tx power reduction will not be enough to clear the interference. On the other hand, when the Tx power of the interfering cells is adjusted (APO), just the three cells are shutdown.

Fig. 5 shows the number of cells that can remain on when the downtilt is modified. In the case of FTO, different downtilt values have been used, from the default $6^{\circ}$ up to $15^{\circ}$, while in the case of ATO, the downtilt of each cell depends on its interference to the RA. In this case, if the FTO downtilt value is higher, the number of cells that can remain on is higher. On the other hand, when lower FTO downtilt values are used, more cells have to be shutdown since the downtilt is not enough to clear the interference.

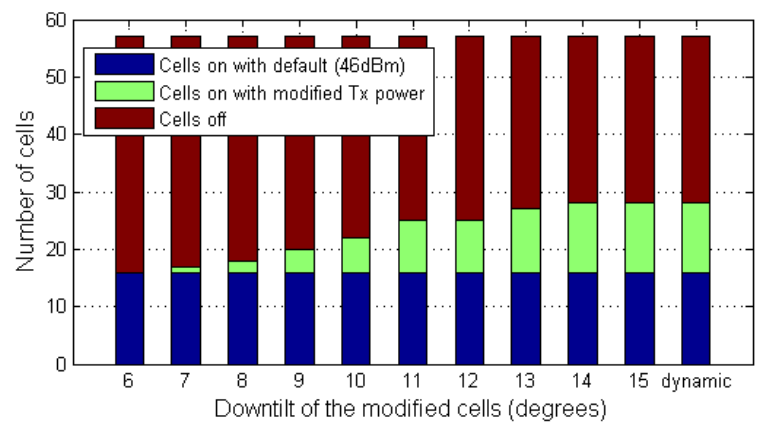

Fig. 5. Downtilt optimization for wireless cameras: Number of cells on, modified and off

In the case of downtilt, there is a maximum attenuation that can be achieved. In the case of ATO, it can be observed that 21 cells out of the 41 interfering cells need to be shutdown independently of the downtilt.

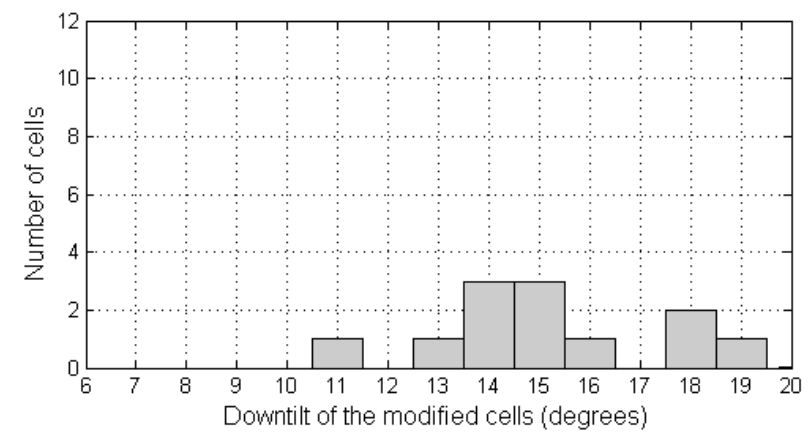

Fig. 6. Histogram of the downtilt for ATO (wireless cameras)

The number of shutdown cells, and the modifications on the Tx power and downtilt have an impact on coverage. As an example, Fig. 7 shows the coverage increase when the APO is applied compared to the scenario without optimization.

Due to the coverage improvement, the average user throughput is likely to improve. Fig. 8 presents a comparison of the average user throughput, when the MNO is not using the ASA resources, when it is using the ASA resources without and with the Reservation Area (as an upper bound), and finally without optimization and for the different types of border 
optimization. It must be highlighted that these results also depend on the size of the Reservation Area.

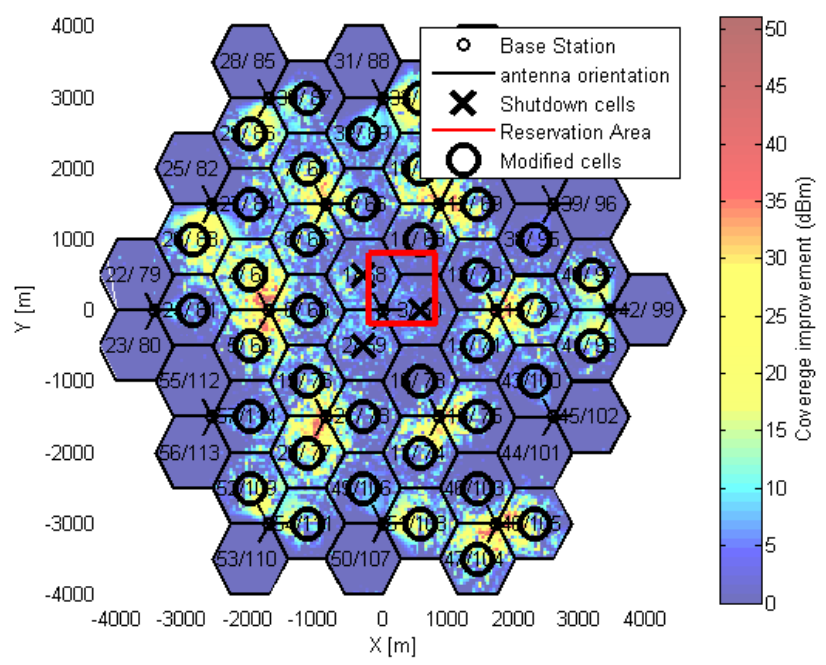

Fig. 7. Coverage improvement map for APO (wireless camera)

The best scenario for the MNO will be when the $2300 \mathrm{MHz}$ frequency band is available at the whole network, without a Reservation Area where the incumbent uses the spectrum. In this case, the average throughput increases around a 30\%. On the other hand, if the spectrum is not available at the RA, there is still a considerable improvement (15\%), even without optimization.

If the MNO uses a border optimization method, the average user throughput increases. The predefined values both for Tx power and downtilt (FPO and FTO), are easier to implement in the network but the average throughput increase is less significant. Adapting each cell independently allows selecting the best value for each case, providing the best optimization.

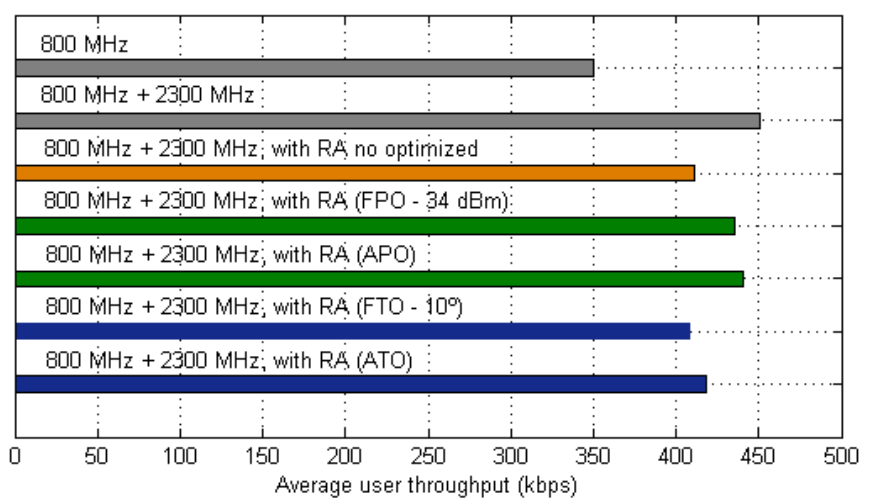

Fig. 8. Average user throughput comparison for wireless cameras

In the case of the downtilt optimization, the average throughput is less significant, since the only way to clear the interference for many cells is the shutdown, due to the limitation of the backward attenuation (at most $20 \mathrm{~dB}$ ). On the other hand, there is no such a fundamental limitation on the Tx power reduction, which is the optimization method with the best average throughput increase (25\% increase for FPO).

\section{B. Cameras on buildings}

The cameras on the top of the buildings receive the signal from the MNO base stations stronger than the wireless cameras located in the ground, due to the difference in height. Due to the higher signal strength, in this scenario, the MNO would not be allowed to use the ASA spectrum at any of the base stations without optimization methods.

In the case of Tx power optimization, Fig. 7 shows that the Tx power should be reduced at least to $37 \mathrm{dBm}$ in the case of FPO, to be able to use the spectrum at some cells without interfering the incumbent. In the case of APO, some of the cells need to be use extremely low (from $37 \mathrm{dBm}$ up to $0 \mathrm{dBm}$ ).

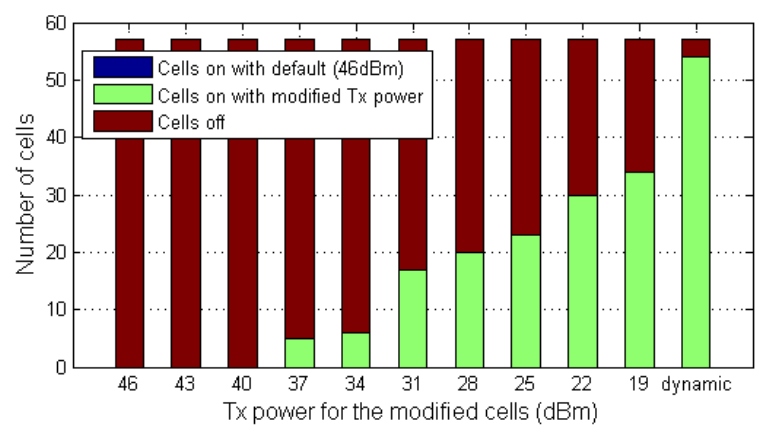

Fig. 9. Txpower optimization for cameras on buildings: Number of cells on, modified and off

Although the Tx power reduction is considerable, Fig. 10 shows that there is still a significant gain in the average user throughput (10\%), similar for APO and FPO.

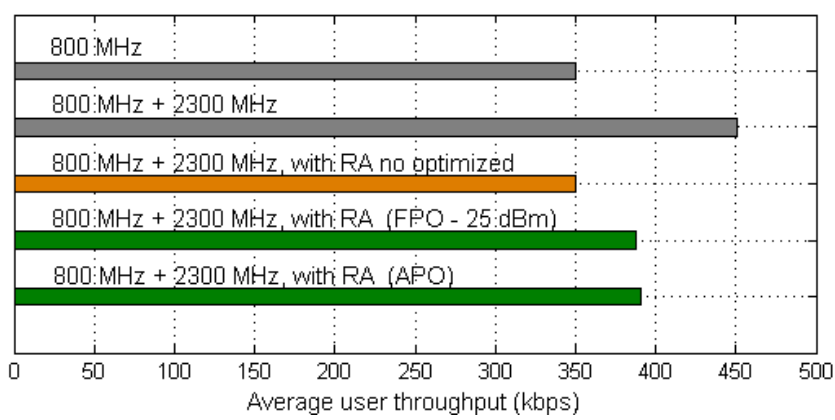

Fig. 10. Average user throughput comparison for cameras on buildings

In this case, since the main lobe of the MNO antenna is likely to be already below the height of most of the cameras, and the interference is exceeding more than the backward attenuation, increasing the downtilt does not bring any benefit.

\section{Video links}

The video links can be mounted in helicopters, motorcycles, pedal cycles, cars, racing cars and boats. In this case, it has been considered that they are mounted in helicopters, which will be the most extreme scenario. In this case, the attenuation losses are even lower than in the case of cameras on the buildings. Therefore, in this particular scenario the MNO would not be allowed to use the ASA spectrum at any of the base stations without optimization method. 
If Tx power optimization is used, Fig. 11 shows that the Tx power should be reduced at least to $28 \mathrm{dBm}$ in the case of FPO, to be able to use the spectrum without interfering the incumbent. In the case of APO, some of the cells need to use extremely low Txpower (from $28 \mathrm{dBm}$ up to $0 \mathrm{dBm}$ ).

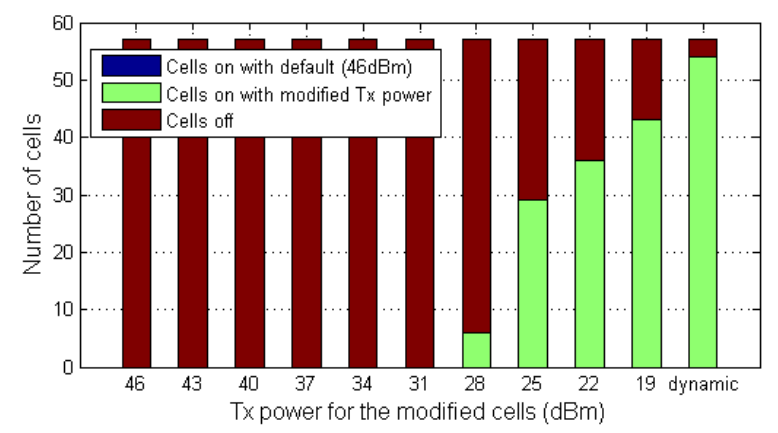

Fig. 11. Txpower optimization for video links: Number of cells on, modified and off

Although the Tx power reduction is considerable, Fig. 10 also shows that there is still a significant gain in the average user throughput (10\%), similar for APO and FPO.

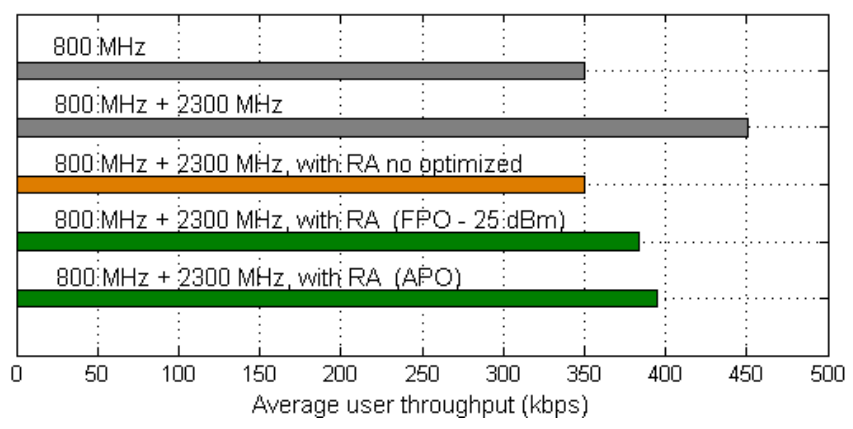

Fig. 12. Average user throughput comparison for video links

\section{CONCLUSIONS}

Authorised Shared Access (ASA) provides a solution to gain access to frequency bands underutilized. Using additional foreign spectrum can bring a lot of benefits to the mobile operator, even if it comes along with certain regulatory and operational restrictions.

If incumbent's victim devices are on comparable locations as the terminals of the mobile operator (e.g. ground floor), it is quite straight forward for the mobile operator to exploit the ASA spectrum as much as possible, whilst respecting the incumbent's restrictions at the same time by analyzing measurements of the own mobile terminals.

The more the locations of the potential victims differ from those of the mobile terminals, the more difficult it becomes for the mobile operator to determine the extent of how ASA spectrum can be used (i.e. which cells are allowed to access the ASA spectrum at which power level and at which antenna tilts), and, consequently the more conservatively and carefully the spectrum usage must be controlled. If the interference is controlled through interference estimations, the existing propagation models (e.g. antenna models) have to be challenged before applying them to ASA interference situations. Alternatively, also tighter collaboration with the incumbent and its equipment can be envisaged. As an example the incumbent may provide interference measurements by its own equipment which can be exploited for enhanced access control.

Most challenging are scenarios with "close-to-free-space" propagation (e.g. helicopter scenario). These scenarios challenge the current antenna designs. However, the spectrum can be used with sufficiently small power, yielding noticeable throughput increase. In our simulations we have reduced the power of macro base station just for ease of studying this effect. In practice, however, it would obviously be more appropriate to deploy small cells with lower antenna heights and small power. Indoor usage of the spectrum might be particularly interesting since the walls will provide additional attenuation towards the victims.

\section{REFERENCES}

[1] "Optimising Spectrum Utilisation towards 2020, NSN White paper," http://nsn.com/file/30301/optimisingspectrum-utilisation-towards-2020, Espoo, 2014.

[2] CEPT, "ECC Report 205 - Licensed Shared Access (LSA)," February 2014.

[3] J. Khun-Jush, P. Bender, B. Deschamps and M. Gundlach, "Licensed shared access as complementary approach to meet spectrum demands.," in ETSI Reconfigurable Radio Systems Workshop, Cannes, 2012.

[4] CEPT, "ECC Report 172 - Broadband Wireless Systems Usage in 2300-2400 MHz," 2012.

[5] P. Fotiadis, M. Polignano, L. Chavarria, I. Viering, C. Sartori, A. Lobinger and K. Pedersen, "Multi-Layer Traffic Steering: RRC Idle Absolute Priorities \& Potential Enhancements," in IEEE Vehicular Technology Conference, Dresden, Spring 2013.

[6] P. Fotiadis, M. Polignano, I. Viering and P. Zanier, "On the Potentials of Traffic Steering in HetNet Deployments with Carrier Aggregation," in IEEE Vehicular Technology Conference, Seoul, 2014.

[7] "Universal Mobile Telecommunications System (UMTS); Radio Frequency (RF) system scenarios," 3GPP Technical Report TR 25.942, Rev. 11.0.0, October 2012.

[8] M. Hata, "Empirical Formula for Propagation Loss in Land Mobile Radio Services," in IEEE Transactions on Vehicular Technology, 1980.

[9] "Evolved Universal Terrestrial Radio Access (E-UTRA); Further Advancements for E-UTRA physical layer aspects," 3GPP Technical Report TR 36.814, Rev. 9.0.0, March 2010.

[10] CEPT, "ECC Report 204 - Spectrum use and future requirements for PMSE," February 2014. 